Population Study

Oral

Abstract ID: 104

\title{
A qualitative study of factors affecting the compliance of hearing protective device usage among factory workers in Kuantan
}

\author{
Ailin Razali | Nor Azlina Abdul Rahman \\ International Islamic University Malaysia
}

Introduction: Occupational noise-induced hearing loss (NIHL) is an acquired cumulative and irreversible hearing impairment which is totally preventable. Literature review concluded that noise hazard control via administrative and engineering methods are difficult to implement for many reasons. Thus, hearing protection device (HPD) is the best compromise to protect workers from the excessive noise. The purpose of this study is to qualitatively explore the factors affecting the HPD compliance among factory workers in Kuantan. Methods: A purposive sampling of twenty four male workers exposed to occupational noise of $85 \mathrm{~dB}$ and more for 8 hour-period was recruited from $15^{\text {th }}$ July to $31^{\text {st }}$ August 2013 to participate in four focus group discussions. The open-ended guided questions were used during the discussions to explore the workers' perspective, knowledge, attitude and behaviour regarding the use of HPD. The data were analyzed using the conventional content analysis and the key themes were elicited in relation to the study, either toward the positive hearing protective behaviours or the factors that act as the barriers against it. Results: From the findings, five themes have emerged with their respective subthemes including perception of noise in the workplace, use of HPD, barriers against the use of HPD, workplace influence and value of hearing. Our studies found that the three main reasons for workers not to wear HPD are discomfort, cumbersome and impediment of speech / other wanted sounds. Conclusions: Effective hearing conservation programme, particularly on the use of HPD should therefore address these factors to ensure good compliance among the workers.

KEYWORDS: hearing protection device, noise exposure 